\title{
Remote monitoring of vital signs in Chronic Heart Failure patients
}

\author{
Anbarasi $K^{1}$, Hari Nandhini $D^{2}$, Kousalyaa $G^{3}$, Sharmila $B^{4}$ \\ Student, Department of EIE, SREC, Coimbatore, India ${ }^{1,2,3}$ \\ Associate Professor, Department of EIE, SREC, Coimbatore, India ${ }^{4}$
}

\begin{abstract}
Majority of the population, especially the people older than 65, suffer from Chronic Heart Failure leading to frequent hospitalizations which results in high health expenditure. So, we have developed a complete and integrated Information and Communication Technology system on LabVIEW platform that enables the doctors to diagnose and monitor the CHF patients from distance. This system measures the heart rate and blood pressure of the patient through heart rate sensor and blood pressure sensor. The output from these sensors are transmitted to the remote system via Bluetooth. It is then transmitted to the Hospital Information System by using LabVIEW tool. This enables the CHF patients to daily collect vital signs at home and automatically send them to the Hospital Information System and it also allows the physicians to monitor the patients from distance and to take timely actions in case of necessity.
\end{abstract}

Keywords: interrupt service routine, systolic pressure, program counter, parity, diastolic pressure, accuracy.

\section{INTRODUCTION}

Chronic heart failure

Chronic heart failure (CHF) represents one of the technology. Bluetooth module is used to transfer the pulse most relevant chronic diseases in all industrialized rate and pressure rates of patients to the remote system. countries, affecting approximately 15 million people in India. It is the leading cause of hospital admission particularly for older adults reaching a prevalence of $1.3 \%$, $1.5 \%$ and $8.4 \%$ in 54-64 years old, $65-74$ years old and 75 years old or older segments respectively.

Current health care model

The current health care model is mostly in- $A$. hospital based and consists of periodic visits. Previous studies pointed out that in patients with a discharge diagnosis of heart failure, the probability of a readmission in the following 30 days is about 0.25 , with the readmission rate that approaches $45 \%$ within 6 months. Hence the current health care model would result in high expenditure.

Proposed model

This work introduces a novel based heart rate and pressure rate monitoring system. PIC16F877A microcontroller is used in this project. The project module uses a portable sensor module which is placed in the patient's body (finger). This method is very much useful for patients who are prone to heart failures. Our module is useful for patients even when they are in some other places other than hospital. It gives immediate indication of low and high blood pressure rates and heart rates beyond certain limits to the patients as well as to the Hospital Information System. In this project, heart rates are monitored by pulse rate sensor which consists of Schmitt trigger and low power op-amp.

Modern heart rate monitors usually comprise of two elements: a chest strap transmitter and a wrist receiver. In early plastic straps, either water or liquid was required to get good performance. Blood pressure rates are monitored by a pressure sensor which consists of testing belt. Many diagnostic and therapeutic devices incorporate pressure sensors made with Piezo-resistive semiconductor
Values are displayed in Liquid Crystal Display (LCD). The pulse rate and blood pressure are transferred to the Hospital Information System by using LabVIEW technology.

\section{OVERVIEW OF THE PROPOSED METHOD}

Hardware description

The overall block diagram of the module as shown in Fig. 1 consists of a heart rate sensor, a blood pressure sensor, an LCD, a buzzer, a microcontroller (PIC 16F877A).

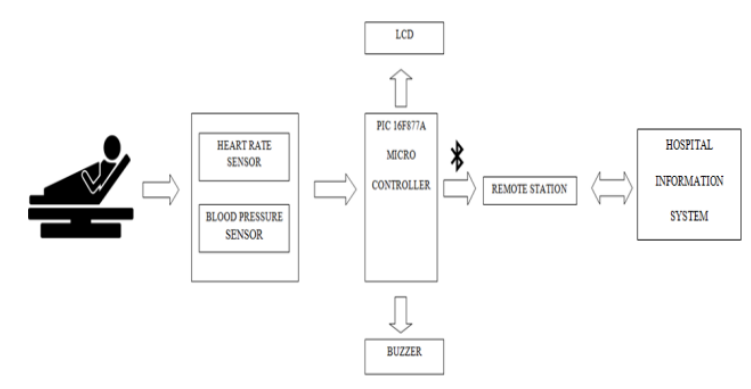

Fig. 1 Overall Block diagram of Remote Monitoring System

The output from the two sensors are sent to the remote station by using a bluetooth module. From the remote station, the data is sent to the Hospital Information System (HIS) by using a Web Publishing tool (LabVIEW).

B. Pressure sensor

The MP3V5050 series piezo-resistive transducer is a state-of-the-art monolithic silicon pressure sensor designed for a wide range of applications, but particularly those employing a microcontroller or microprocessor with A/D inputs. This patented, single element transducer combines advanced micromachining techniques, thin-film metallization, and bipolar processing to provide an 
accurate, high level analog output signal that is proportional to the applied pressure.

\section{Heart rate sensor}

Modern heart rate sensor used in this project comprises of two elements: a chest strap transmitter and a wrist receiver. In early plastic straps, either water or liquid was required to get good performance.

\section{PIC microcontroller}

The PIC 16F877A features 256 bytes of EEPROM data memory, self programming, two comparators, 8 channels of 10 bit Analog-to-Digital(A/D) converter, two capture/ compare/ PWM functions, the synchronous serial port can be configured as either three wire Serial Peripheral Interface or the two wire InterIntegrated Circuit bus and a Universal Asynchronous Receiver Transmitter. All of these features make it ideal for more advanced level A/D applications in automotive, industrial appliances and consumer applications. The PIC 16F877A consists of 21 pins and 5 ports namely: A, B, C, D, E.

\section{MEASUREMENT AND TRANSMISSION}

\section{A. Blood pressure measurement}

Usually when the doctor measures the patient's blood pressure, he will pump the air into the cuff and use the stethoscope to listen to the sounds of the blood in the artery of the patient's arm. At the start, the air is pumped to be above the systolic value. At this point, the doctor will hear nothing through the stethoscope. After the pressure is released gradually, at some point, the doctor will begin to hear the sound of the heart beat. At this point, the pressure in the cuff corresponds to the systolic pressure. After the pressure decreases further, the doctor will continue hearing the sound (with different characteristics). And at some point, the sounds will begin to disappear. At this point, the pressure in the cuff corresponds to the diastolic pressure.

To perform a measurement, a method called oscillometry is used. The air will be pumped into the cuff to be around $20 \mathrm{mmHg}$ above average systolic pressure (about $120 \mathrm{mmHg}$ for an average). After that the air will be slowly released from the cuff causing the pressure in the cuff to decrease.

As the cuff is slowly deflated, the tiny oscillation is measured in the air pressure of the arm cuff. The systolic pressure will be the pressure at which the pulsation starts to occur. The MCU is used to detect the point at which this oscillation happens and then record the pressure in the cuff. Then the pressure in the cuff will decrease further. The diastolic pressure will be taken at the point in which the oscillation starts to disappear.

Safety in design

Since the system developed is a medical instrumentation device, the safety of the user is the first concern to us. The cuff while driven by a 5 volt motor can squeeze the arm really hard and cause injury if being used improperly. So in our device we have 3 levels of security, making sure that the operation can be aborted by the user at anytime. For the first safety design, the microcontroller is programmed in the way such that if the pressure in the cuff is greater than $160 \mathrm{mmHg}$, the motor will stop. For most people, the pressure at $160 \mathrm{mmHg}$ will only cause a time. little discomfort to the arm. This design makes sure that the pressure inside the cuff will never exceed the maximum limit of $160 \mathrm{mmHg}$. The second safety design is to provide an emergency button for the user. While the motor is pumping and the cuff is being inflated, if the user encounters too much discomfort or pain, he/she can press this button to stop the operation immediately. The motor will be stopped and the valve will be opened to release the air out of the cuff.

However, we still think that only a pushbutton is not enough for the safety of the user. This is because the emergency button still relies on the operating system of the program in the MCU. This switch must be able to disconnect the circuit from the power supply immediately. This way it is certain that the user will be able to stop the operation even when the emergency button does not work. Heart rate measurement

This implementation of a heart monitor involves low cost amplifier and filter components coupled with a sophisticated microcontroller and LCD screen. Because the device is most useful if it is portable it was designed with use of one or two $9 \mathrm{~V}$ batteries. The amplifier and filter stage of the implementation were successful with an ECG successfully detected and recorded. In doing this the output voltage was found to be strongly related to the quality of contact between the electrodes and the skin and was observed to be highly variable. This complicated the calculation and display of the actual rate since the proposed microcontroller code relied on a fixed reference which the peak of the ECG would cross and trigger the timer.

The variability of the voltage output made this approach unfeasible. Using a fixed signal made demonstration of this part of the circuit possible. This project successfully implemented an ECG while the Digital Heart Rate counter was only partially successful. The weak signal heart rate signal was amplified in the presence of noise from other muscles and electrical sources but we were unable to create an integrated device which could take this signal and calculate the heart rate. The heart is one of the most vital organs within the human body. It acts as a pump that circulates oxygen and nutrient carrying blood around the body in order to keep it functioning. The circulated blood also removes waste products generated from the body to the kidneys. When the body is exerted the rate at which the heart beats will vary proportional to the amount of effort being exerted. By detecting the voltage created by the beating of the heart, its rate can be easily observed and used for a number of health purposes. An electrocardiogram (ECG) is a graphical trace of the voltage produced by the heart. There are 5 identifiable features in an ECG trace which corresponds to different polarisation stages that makes up a heartbeat. These deflections are denoted by the letters $\mathrm{P}, \mathrm{Q}, \mathrm{R}, \mathrm{S}$ and $\mathrm{T}$. By detecting the $R$ peaks and measuring the time between them the heart rate can be calculated and then displayed. A persons heart rate before, during and after exercise is the main indicator of their fitness. Measuring this manually requires a person to stop the activity they are doing in order to count the number of heart beats over a period of 
INTERNATIONAL JOURNAL OF INNOVATIVE RESEARCH IN ELECTRICAL, ELECTRONICS, INSTRUMENTATION AND CONTROL ENGINEERING Vol. 3, Issue 3, March 2015

\section{Calculating heartbeat rate}

The number of heart beats per minute is calculated using a three beat average. Two variables in the $\mathrm{C}$ main function, counter and pulse period, accurately track the time scale. Each output sample from the QRS discriminator is compared against a set threshold to detect the presence of a beat. Pulse period is incremented by one during every sample period. Because each sample occurs every $1 / 512$ second, it is easy to track the time scale based on the number of counts in the pulse period variable.

A 128-sample time window is used as a denounce time using counter. Every time a beat is detected, counter is reset and the LCD icon with four arrows is turned on to represent the heart beat. If a beat is not detected for 128 consecutive samples, a separation between successive beats is identified and the LCD icon with four arrows is turned off. The pulse period is accumulated for three consecutive beats. On the third beat, pulse period is used for the calculation of heart-rate per minute and reset.

Heartbeat rate per minute $=1 /$ [pulse period/ $(3 \times 512 \times$ 60) $]=92160 /$ pulse period

\section{Processing \& display}

Due to the use of a microcontroller to calculate the beats per minute (BPM), it was decided that a liquid crystal display (LCD) module would be the most flexible way of displaying this numerical output. It was originally planned that several seven segment displays could be used, but again it was deemed worthwhile to integrate the display unit together and limit the number of components required. In addition, the information which the LCD could convey was greater.

Regarding the actual BPM calculation (assuming that it was possible to translate each $\mathrm{R}$ part (the blip/spike peak) into singular events occurring in a timely fashion of course) it was originally going to be done by measuring the total number of spikes within a certain amount of time and then multiplying this count by a factor (as it is done when using a clock and your hand). With the use of the microcontroller however more precise measurements were able to be made resulting in an output of greater accuracy and speed.

\section{Transmission of signals}

In this project, the code is embedded into the microcontroller using MPLab software and is compiled using Hightech $\mathrm{C}$. The heart rate sensor used in this project outputs a digital value. Hence unlike pressure sensor there is no need of any Analog to Digital Conversion. Bluetooth module is used to transfer signals from the sensor module to the remote system.

The heart beat rate and the blood pressure measured from the patients are transmitted to the LCD unit, where the values are displayed. When these values exceed the threshold value the buzzer gets switched ON. The Web Publishing Tool dialog box is shown in the Fig. 2.

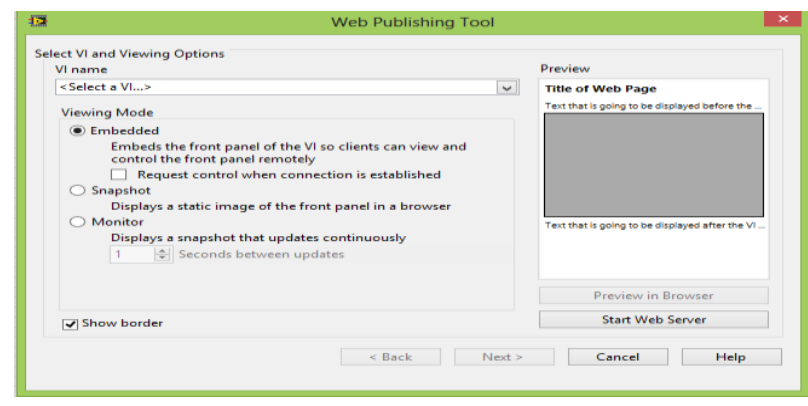

Fig. 2 Web Publishing Window

Web Publishing Tool (LabVIEW platform) is used to transfer the signals from the remote system to the Hospital Information System.

The desired display format in HIS can be obtained using the Web publishing Tool dialog box shown in Fig.2 and also this tool is user-friendly when compared to other transmission tools available in LabVIEW.

A.

IV. RESULTS AND DISCUSSION Project setup

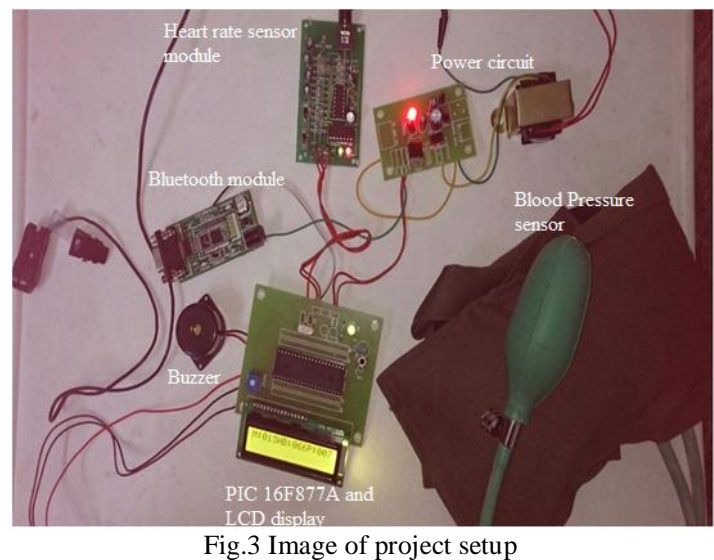

The entire project set up is shown in the Fig.3.The heart rate and the blood pressure sensors are connected to the respective ports of the microcontroller as discussed earlier. The output from the sensors is transmitted via Bluetooth module which is also connected to the microcontroller.

The step down transformer shown in the Fig. 3 converts the 230 volt $\mathrm{AC}$ to 12 volt $\mathrm{AC}$ and the power circuit shown in the Fig. 3 consists of a full wave rectifier which provides a rectified DC supply of 5 volts to all the components.

\section{B. Result before bluetooth transmission}

The measured heart beat rate and blood pressure are displayed in the Liquid Crystal Display which is shown in the Fig.4 in which M represents the count of the timer, $\mathrm{HB}$ indicates heartbeat rate and $\mathrm{P}$ represents the blood pressure

For every 60 seconds the heart beat rate $(\mathrm{HB})$ is displayed while the blood pressure rate is displayed continuously regardless of the 60 seconds count. 
INTERNATIONAL JOURNAL OF INNOVATIVE RESEARCH IN ELECTRICAL, ELECTRONICS, INSTRUMENTATION AND CONTROL ENGINEERING Vol. 3, Issue 3, March 2015

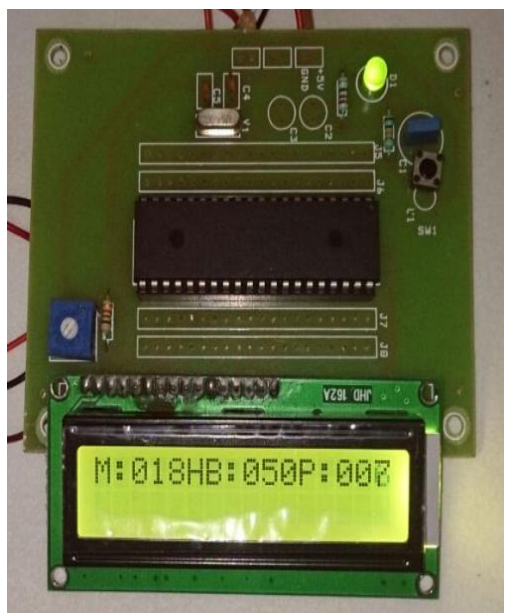

Fig. 4LCD display

\section{Transmission results}

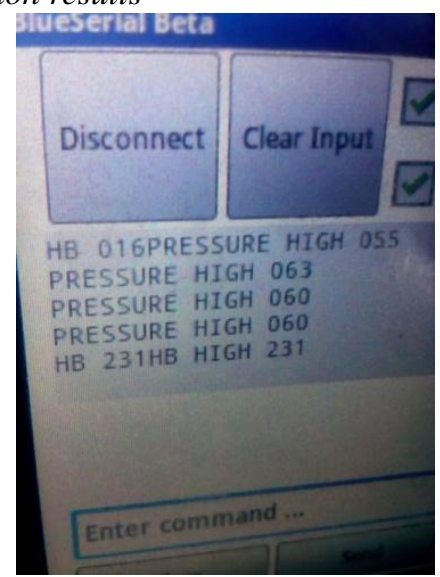

Fig. 5 Result after transmission

The measured data are sent to the remote system and displayed as shown in the Fig.5. The result as shown in the Fig. 5 indicates whether the pressure is high or low by comparing with the threshold value that has been set. When the heart beat rate and blood pressure goes beyond the threshold value the buzzer gets switched ON. The obtained result can be displayed in the Hospital Information System after transmission and can be viewed by the doctor. The doctor then analyses the report and takes the necessary action. Also the doctor's feedback about the patient's health is sent to the remote system.

\section{CONCLUSION AND FUTURE SCOPE}

\section{A. Conclusion}

Finally the blood pressure ratings and heart rates can be measured using the required sensors by making it as a portable device and provide it to the patients. It is very much useful to the patients those who are away from hospitals. This system is affordable. Thus by using this system we can prevent frequent hospitalizations of the heart patients is prevented.

\section{B. Future scope}

The entire module is to be made handy for the convenience of the patients. The accuracy of the sensors is also to be improved. Devices to measure additional parameters such as SPO2, weight, blood sugar level are to be included.

\section{REFERENCES}

[1] Mohd Fadlee A. Rasid and Bryan Woodward, "Bluetooth telemedicine processor for multichannel biomedical signal transmission via mobile cellular networks," IEEE Trans. Inf. Tech in Biomedicine, vol .9,no.1, 2005 pp.35-43.

[2] N.Daja, I.Relin, “ Telemonitoring in cardiology-ECG Transmission through mobile phones," J.Annals Academy Studenica,vol4,2001, pp.63-66.

[3] P.Bauer,M.Sichitiu, R.Istepnaian and K.Premaratne , "The Mobile Patient Wireless Distributed Sensor Networks for patient monitoring and care," in Proc. IEEE EMBS International Confrence on Information Technology Applications in Biomedicine, Arlington,pp.17-21,2000.

[4] Jordan,R.Abdallah and C.T., " Wireless and Networking An Overview" IEEE Antennas and Propagation Magazine, vol 44 Issue:1,pp.185-193,Feb 2002.

[5] Jimena Rodriguez, Alfredo Goni, and Arantza Illaramendi, "Realtime classification of ECGs on a PDA," IEEE Trans inf.Tech in Biomedicine, vol.9.no.1,2005,pp 23-34.

[6] B.Woodward, R.S.H. Istepanian and C.I.Richards, “ Design of Telemedicine system using a mobile Telephone," IEEE Trans.inf.Tech in Biomedicine,vol.5,no.1, march 2001,pp 13-15.

[7] C. Berry, D.Murdoch and J.McMurray, "Economics of Chronic eart Failure,” Eur. J. Heart fail., vol. 3, no. 3,pp. 283-291, june 2001.

[8] F. Zannad, N. Agrinier, and F. Alla, "Heart failure burden and therapy," Europace, vol. 11, no. 5, pp. 1-9, Nov, 2009.

[9] E. Seto, "Cost comparison between telemnitoring and usual care of heart failure: A systematic reviw," Tltmed. J. E-Health, vol. 14, no. 7,pp. 679-686, Sep. 2008.

[10] C. Klersy, A. De Silvestri, G. Gabutti, F. Regoli, and A. Auricchio, "A meta-analysis of remote monitoring of heat faoliure patients," J. Amer. Coll. Cardiol., vol. 54, no .18, pp. 1683-1694, Oct. 2009. 